\title{
Erreurs médicales, excuses et lois sur les excuses
}

$\mathrm{D}$ ans la vie de tous les jours, lorsqu'il se produit une erreur, on s'attend à ce qu'il y ait divulgation, excuses et dédommagement. En soins de santé, lorsqu'il se produit une erreur médicale, la crainte de poursuites pour faute professionnelle l'emporte souvent sur la divulgation et les excuses.

La divulgation intégrale au patient constitue la solution responsable sur les plans éthique et professionnel. Elle est aussi vitale pour l'amélioration de la sécurité des patients et de la qualité des soins ${ }^{1}$. En ne divulguant pas les événements indésirables, le médecin manque d'honnêteté, d'ouverture et de respect envers le patient. La non-divulgation peut de plus exposer le patient à des préjudices futurs parce qu'il ne sait pas ce qui s'est produit. La divulgation fournit au patient des renseignements qui peuvent jouer un rôle vital dans des décisions et des choix à venir en soins de santé. La franchise entre collègues au sujet des erreurs est aussi cruciale pour l'apprentissage professionnel, l'amélioration de la sécurité des patients et la confiance du public envers le système de santé1.

Il est important de présenter des excuses en même temps que l'on divulgue une erreur médicale. Pour s'excuser, il faut reconnaître l'événement et le rôle qu'on y a joué, et exprimer un regret sincère à l'égard de l'état du patient. Des excuses peuvent avoir de profonds effets guérisseurs pour toutes les parties. Elles peuvent aider à atténuer les sentiments de culpabilité et de honte chez le médecin. Chez le patient, elles peuvent faciliter le pardon et jeter les bases d'une réconciliation².

Les lois sur les excuses, cherchant à accommoder les divergences entre les impératifs d'éthique et de sécurité liés à la divulgation et aux excuses et l'instinct puissant qui pousse à garder le silence par crainte de poursuites pour faute professionnelle, ont été conçues pour atténuer les inquiétudes face aux répercussions juridiques de la divulgation et des excuses. Ces mesures ont fait leur apparition aux États-Unis au cours des années 1990 dans le contexte des efforts déployés pour améliorer la déclaration des erreurs médicales et la sécurité des patients. Depuis, les médecins et les hôpitaux sont devenus plus transparents, honnêtes et ouverts en expliquant rapidement les résultats imprévus. Conjuguée aux offres rapides de règlement présentées par les hôpitaux, cette attitude a entraîné une diminution spectaculaire du nombre des poursuites pour faute professionnelle ${ }^{3}$. La contribution réelle des excuses et des lois afférentes à ce résultat est toutefois moins claire parce qu'il s'agit là de deux éléments des efforts réglementaires et institutionnels de plus grande envergure déployés pour surmonter le problème complexe du silence entourant les erreurs médicales. De plus, le dédommagement et le règlement rapide peuvent constituer un élément moteur important de la réduction du nombre des poursuites ${ }^{4}$. Dans les pays dotés d'un régime d'indemnisation sans égard à la faute, comme la Suède et la France, il y a moins de plaintes.

La Constitution canadienne attribue aux provinces et aux territoires la compétence en matière de responsabilité civile. La ColombieBritannique $^{5}$ et la Saskatchewan ${ }^{6}$ ont adopté la première loi canadienne sur les excuses en 2006 et le Manitoba a suivi en $2008^{7}$. L'Ontario et l'Alberta ont depuis présenté des mesures législatives semblables ${ }^{8}$. La protection accordée par les lois sur les excuses est semblable dans toutes les administrations canadiennes.

On a pu affirmer qu'il n'est pas nécessaire d'avoir une loi sur les excuses pour que les travailleurs de la santé s'acquittent de leur responsabilité professionnelle en matière de divulgation et qu'à cause de ces lois, il sera plus difficile d'intenter des poursuites fructueuses pour faute professionnelle ${ }^{9}$. Il semble toutefois improbable que des excuses protégeraient les médecins dans les cas de négligence grossière. De plus, selon l'Association canadienne de protection médicale, dans les administrations qui n'ont pas de loi sur les excuses, «les médecins doivent savoir que la présentation d'excuses et toute admission de faute ayant été faite en cette occasion peuvent être admissibles dans une action en justice ou une affaire déposée devant le Collège concernant un événement indésirable» ${ }^{10}$. Cet énoncé qui risque de refroidir les excuses et d'appuyer un code du silence porte à croire que les lois sur les excuses joueront un rôle important dans les conseils donnés aux médecins devant faire face à une erreur médicale ou à un événement indésirable et aux poursuites qui en découlent.

Divulguer une erreur et s'en excuser en tout sincérité n'est ni simple ni indolore. Les médecins et autres travailleurs de la santé ont besoin d'une formation sur la meilleure façon de procéder après une erreur médicale ou un événement indésirable. Ils doivent aussi mieux connaître leurs obligations légales, éthiques et professionnelles en la matière. Les erreurs exigent une réaction capable de répondre simultanément aux besoins du patient, du travailleur de la santé et du système. Comme les conseils de l'Association canadienne de protection médicale tendront à décourager les excuses, il faudra insister pour que les provinces et des territoires qui ne l'ont pas encore fait s'empressent d'adopter des lois en la matière.

\section{Noni MacDonald MD MSc}

Rédactrice de section, Santé publique et santé des populations Amir Attaran LLB DPhil

Rédacteur associé, Éditoriaux

$J A M C$

Avec l'équipe de rédaction de l'éditorial (Paul C. Hébert MD MHSc, Matthew B. Stanbrook MD PhD, Barbara Sibbald BJ, Ken Flegel MDCM MSc).

Intérêts concurrents : Voir www.cmaj.ca/misc/edboard.shtml.

Traduit par le Service de traduction de l'AMC.

\section{RÉFÉRENCES}

1. Kaldjian LC, Jones EW, Rosenthal GE, et al. An empirically derived taxonomy of factors affecting physicians' willingness to disclose medical errors. J Gen Intern Med 2006;21:942-8.

2. Lazare A. Apology in medical practice: an emerging clinical skill. JAMA 2006;296:1401-4.

3. Geier P. Emerging med-mal strategy: «I'm sorry.» Natl Law J 2006;July 24. Disponible : www.law.com/jsp/article.jsp?id=900005550355 (consulté le 5 décembre 2008).

4. Dresser R. The limits of apology laws. Hastings Cent Rep 2008;38:6-7.

5. Apology Act, SBC 2006, c 19. Disponible : www.qp.gov.bc.ca/statreg/stat/a /06019_01.htm (consulté le 5 décembre 2008).

6. Evidence Act, SS 2006, c E-11.2, s 23.1(1). Disponible : www.canlii.org/sk/laws /sta/e-11.2/20080818/whole.html (consulté le 5 décembre 2008).

7. Apology Act, SM 2008, c 42. Disponible : http://web2.gov.mb.ca/laws/statutes /ccsm/a098e.php (consulté le 5 décembre 2008).

8. Silversides A. Apology measures proliferate. CMAJ 2008;179:1115-6.

9. Bailey TM, Robertson EC, Hegedus G. Erecting legal barriers: new apology laws in Canada and the patient safety movement: useful legislation or a misguided approach? Health Law Can 2007;28:33-8.

10. Association canadienne de protection médicale. La législation canadienne sur les excuses : Qu'est-ce que cela signifie pour les médecins? [document d'information]. Ottawa (Ont.) : L’Association; 2008. Disponible : https://www4.cmpa-acpm .ca/cmpapd04/docs/resource_files/infosheets/2008/com_is0889-f.cfm (consulté le 10 décembre 2008). 